\title{
Modeling of sedimentation and filtration layer formation by discrete element method
}

\author{
E. Dyachenko ${ }^{1, a}$, J. Dueck ${ }^{2, b}$ \\ ${ }^{1}$ Tomsk State University, \\ Prospekt Lenina, 36, Tomsk, 634050, Russia \\ ${ }^{2}$ University of Erlangen Nuremberg, \\ Erlangen Paul-Gordan-Straße, 3, D-91052, Germany \\ E-mail: ${ }^{a}$ Evg.Dya@gmail.com, ${ }^{\mathrm{b}}$ Johann.dueck@mbt.uni-erlangen.de
}

Получено 17 ноября 2011 г.

\begin{abstract}
The numerical model of sedimentation and suspension filtration is proposed in this paper. The model is based on dynamic variant of discrete element method. This model represents the particles behavior on microand meso-scales: pores, arches, flocks formation. In addition, the proposed model qualitatively reproduces macro phenomenon: sedimentation of particle layer, slow shrinkage of the layer, sealing of the layer under its own weight of the particles and the external applied force.
\end{abstract}

Keywords: sedimentation layer formation, filtration cake consolidation, discrete element method

\section{Моделирование образования седиментационного и фильтрационного слоев методом дискретных элементов}

\section{Е. Н. Дьяченко ${ }^{1}$, И. Г. Дик ${ }^{2}$}

${ }^{1}$ Томский государственный университет, Россия, 634050, г. Томск, пр. Ленина, 36

${ }^{2}$ Университет Эрланген-Нюрнберг, Германия, D-91052, Эрланген, Пауль-Гордан-Штрассе, 3

В работе предлагается численная модель седиментации и фильтрования суспензии, основанная на динамическом варианте метода дискретных элементов. Эта модель отражает поведение частиц на микро- и мезоуровне: образование пор, арок, хлопьев. Кроме того, предложенная модель качественно воспроизводит макроэффекты: осаждение слоя частиц, медленные процессы усадки этого слоя, уплотнения слоя под действием собственного веса частиц и приложенной извне силы.

Ключевые слова: образование седиментационного слоя, консолидация фильтрационного слоя, метод дискретных элементов

Citation: Computer Research and Modeling, 2012, vol. 4, no. 1, pp. 105-120.

This research work is carried out within the framework of Federal Target Program "Research and scientific-pedagogical cadres of Innovative Russia" in 2009- 2013 years (State contract number P26 on 25/03/2010). 


\section{Introduction}

Layers of dispersed material exist not only in nature, e.g. as sediment, but also in technical processes such as water purification or phases separation by sedimentation and filtration [Zhuzhikov, 1971; Wakeman, Tarleton, 1999]. Density of sediment mainly determines not only the moisture content, but also the mechanical characteristics of the layers, such as their mobility.

Modeling layer formation by methods of continuum mechanics doesn't allow taking into account events that occur at the micro level - the level of individual particles and pores. Physical and chemical factors, such as the effects arising due to surface charge, hydrophobicity / hydrophilicity of suspension particles or a filter layer, is also absent in the classical models of layers formation of dispersed materials.

The dynamic method discrete element (DEM) was selected as the main tool for numerical simulation of the layers. Theoretical foundations of DEM and the review of applications can be found in [Zhu et. al., 2007; Zhu et. al., 2008]. This method has fundamental differences from the approaches of continuum mechanics. Within the DEM the behavior of particles is simulated at the micro and meso level, i.e. the motion of individual particles and the formation of mesostructures (pores, arches, flakes). In addition, the proposed model qualitatively reflects macro features: sedimentation of particles, slow process of layer shrinkage, a layer sealing of under its own particles weight and the applied external force.

Due to the fact that the problem is rather complex: there is a huge number of particles in consideration, a variety of physical interaction forces between particles and between particles and the medium - numerical solution is feasible only on modern cluster computers.

Different to the static method of discrete elements used for example in [Dueck, Ugov, 2005; Dueck, Dyachenko, Minkov, 2006; Neesse, Dueck, Djatchenko, 2009], the dynamic version of the method is able to directly simulate the transient effects of particles collective behavior, such as shrinkage of the layer.

\section{Mathematical statement}

The mathematical statement of the problem is formulated in terms of the discrete element methods. It is supposed that for each spherical particle of the collective is solved the system of motion equations for the center of mass:

$$
\left\{\begin{array}{l}
\frac{d \mathbf{v}_{i}}{d t}=\frac{\mathbf{F}_{i}}{m_{i}} \\
\frac{d \mathbf{x}_{i}}{d t}=\mathbf{v}_{i},
\end{array}\right.
$$

where $m_{i}=\rho \frac{4}{3} \pi r_{i}^{3}$ - particle mass; $t$ - time, $\mathbf{F}_{i}-$ the resulting force acting on the particle; $\mathbf{x}_{i}-$ the coordinate of particle center of mass; $\mathbf{v}_{i}$-the particle velocity; $\rho$-density of material of the particle.

It is supposed that in some moment of time the particle $i$ has $N_{i}$ contact point with other particles or with the boundary of the computational domain.

The force $\mathbf{F}_{i}$ is written as

$$
\mathbf{F}_{i}=\sum_{j=1}^{N_{i}}\left[\mathbf{f}\left(\mathbf{x}_{i}, \mathbf{x}_{j}, r_{i}, r_{j}\right)+\mathbf{F}_{\mathrm{ad}}\left(r_{i}, r_{j}\right)+\mathbf{F}_{\mathrm{fr}, i j}\right]+\mathbf{g} m_{i}+\mathbf{F}_{\mathrm{st}, i},
$$

where $N_{i}$ - the number of contacts of the particle with radius $r_{i}$ with the neighboring particles with radii $r_{j}, \mathbf{g}$-acceleration of gravity;

$\mathbf{f}\left(\mathbf{x}_{i}, \mathbf{x}_{j}, r_{i}, r_{j}\right)$ - elastic force of interaction between particles $i$ and $j$; 
$\mathbf{F}_{\mathrm{ad}}\left(r_{i}, r_{j}\right)$ - adhesion force between two particles;

$\mathbf{F}_{\mathrm{f}, i j}$ - full sliding and rolling friction between two particles;

$\mathbf{F}_{\mathrm{st}, i}$ - resistance force, calculated by Stokes' law.

It's supposed that the forces which are out of sum affect also in absence of any contacts.

\section{Elastic force of particle interaction}

$\mathbf{f}\left(\mathbf{x}_{i}, \mathbf{x}_{j}, r_{i}, r_{j}\right)$ can be found by solving the Hertz problem [Timoshenko, Godier, 1951; Landau, Livshic, 1987] for hard spheres. In this problem interaction of two spheres are considered as shown in Fig. 1.

The solution of Hertz problem gives the relation between deformation of the particles $h$ and applied force $f: f=K h^{\frac{3}{2}} R^{\frac{1}{2}}$, where $f$-absolute value of the particles interaction forces, $K=\frac{2}{3}\left(\frac{E}{1-v^{2}}\right)-$ effective Young's modulus, $E$ - Young's modulus и $v$ - Poisson's ratio of particle material, $R=\frac{2 r_{i} r_{j}}{r_{i}+r_{j}}-$ effective radius of two particles, $h-$ penetration of particles.

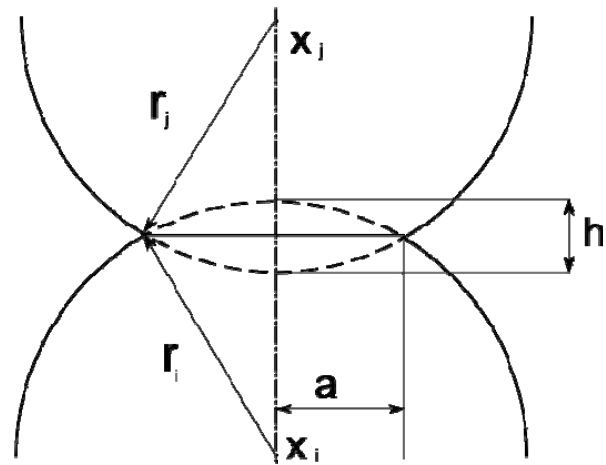

Fig. 1. Relationship between the radius of contact area $a$ and the depth of deformation $h$

To account for the dissipation of energy in the process of interaction between two particles the energy restitution coefficient $B$ is introduced, which varies from 1 (the particle completely restores the shape; energy dissipation is absent) to 0 (the particle doesn't restore the shape; energy is completely transformed in plastic deformation).

In addition, the interaction force between the particles depends on whether the particles are approaching or moving apart:

$$
f=\left\{\begin{array}{l}
K h^{\frac{3}{2}} R^{\frac{1}{2}}, \frac{d h}{d t}>0 \text { (approaching), } \\
B K h^{\frac{3}{2}} R^{\frac{1}{2}}, \frac{d h}{d t}<0 \text { (moving apart). }
\end{array}\right.
$$

Let's consider a particle that had kinetic energy $W_{0}$ before the interaction with other particles. This initial kinetic energy is converting into the potential energy: $W_{p}=\int_{0}^{h_{\max }} f d h=\frac{2}{5} K R^{\frac{1}{2}} h_{\max }{ }^{\frac{5}{2}}$. Then the particles are moving apart and the energy is converting to kinetic one again: $W=-\int_{h_{\max }}^{0} f d h=\frac{2}{5} K B R^{\frac{1}{2}} h_{\max }{ }^{\frac{5}{2}}$. The ratio of the particle energy after the interaction to the energy before the interaction is the energy restitution coefficient: $W / W_{0}=B$. 
Thus, the particle interaction force written in the form (3) meets the conditions of elastic deformation with the loss of energy. Such a form of particles interaction forces is used in [Moysey P. A., Thompson, 2005; Ananda et. al., 2008; Vu-Quoc, Zhang, Walton, 2000]. Residual plastic deformation is not considered. Model with an energy restitution coefficient is called in the literature a hysteresis model [Kruggel-Emden et. al., 2007].

Weight of overlying particles is taken into account automatically. In case any new particle settles on the already-formed layer the elastic force of ground reaction arises between the particle and the layer. This force can bring out of balance the layer particle which is in contact and it will move in the direction of addition force - down. Due to this shift the additional force arouses between the displaced particles and surrounded particles. Then the stress wave and small movements of the particles will distribute to the boundaries of the computational domain. The extra stress will occur with each of new settled particles in the layer so that the sum of all stresses will be the overlying layer weigh.

\section{The adhesion force}

During the interaction of microparticles there are the elastic forces of the material in direct contact and forces of surface interaction, the role of which increases with decreasing particle size.

The adhesion force Fad consists of attractive and repulsive components [Israelachvili, 1995]: $F_{\text {ad }}=F_{\text {at }}+F_{\mathrm{r}}$, where $F_{\text {at }}=\frac{A}{6 H_{0}^{2}} R$ - attractive components due to the Van der Waals force, $F_{\mathrm{r}}=-\frac{4 \pi z^{2} e^{2}}{k T} \psi_{0}{ }^{2} \frac{c_{\infty}}{\kappa} \exp \left(-\kappa H_{0}\right) R-$ repulsive component according the Gouy-Chapman theory, A Hamaker's constant, $c_{\infty}$ - ions concentration in liquids away from the particle surface, $e-$ electro charge, $H_{0}$ - distance between the surfaces of interacting particles (usually the intermolecular distance), $k$ - Boltzmann's constant, $z$ - valence of medium ions; $\psi_{0}$ - surface potential of particles, $T-$ temperature, $\frac{1}{\kappa}=\sqrt{\frac{\varepsilon \varepsilon_{0} k T}{e^{2} c_{\infty} z^{2}}}-$ wavelength Debay.

Thereby the adhesion force can be written as:

$$
F_{\text {ad }}=\left(\frac{A}{6 H_{0}^{2}}-\frac{4 \pi z^{2} e^{2}}{k T} \psi_{0}^{2} \frac{c_{\infty}}{\kappa} \exp \left(-\kappa H_{0}\right)\right) R=K_{\text {ad }} R,
$$

where $K_{\mathrm{ad}}=\frac{A}{6 H_{0}{ }^{2}}-\frac{4 \pi z^{2} e^{2}}{k T} \psi_{0}{ }^{2} \frac{c_{\infty}}{\kappa} \exp \left(-\kappa H_{0}\right)-$ specific surface energy of adhesion forces or the adhesion coefficient, which depends on the physical and chemical characteristics of the system. Kad can be varied by changing of ions concentration $c_{\infty}$ in the solution by adding a certain amount of salts (eg, $\left.\mathrm{MgCl}_{2}, \mathrm{AlCl}_{3}\right)$.

\section{Friction force}

To simplify the consideration we introduce a generalized friction force and a generalized friction coefficient, whose value lies in the range between the coefficient of rolling friction and coefficient of sliding friction. In the case of simulation of particles significantly different from spheres, the value of the generalized friction coefficient is close to the value of the coefficient of sliding friction, otherwise the value will be significantly shifted in the direction of rolling friction coefficient. This approach allows us to avoid the consideration of the equation of force moments.

Let's consider the frictional force acting on the particle $i$ in contact with particle $j$ (particle in rest relative to each other), with some forces acting at particle $i$ and tending to unbalance it (Fig. 2). 


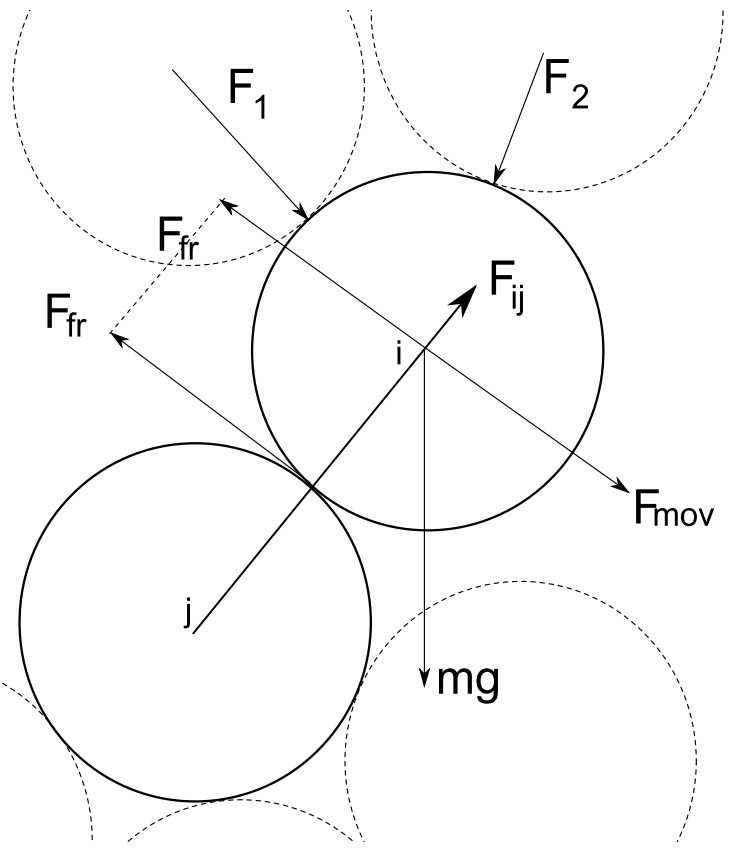

Fig. 2. The scheme of acting forces

The force of friction in the contact of two particles at rest is a bilinear function of the moving forces that can be written in vector form (taking into account that the direction of the vector of the friction force is opposite to the vector the moving force):

$$
\mathbf{F}_{\mathrm{fr}}=\left\{\begin{array}{c}
-\mathbf{F}_{\mathrm{mov}}, F_{\text {mov }} \leq F_{\text {frMax }}, \\
-F_{\text {frMax }} \frac{\mathbf{F}_{\text {mov }}}{\left|\mathbf{F}_{\text {mov }}\right|}, F_{\text {mov }}>F_{\text {frMax }},
\end{array}\right.
$$

where $F_{\text {mov }}=\sum F_{i}+m g-$ moving force - the result of all forces except friction force in current contact, $F_{\mathrm{fr} \text { ax }}=k_{\mathrm{fr}}|\mathbf{f}|-$ the maximum value of the friction force.

In case when particles are moving relative to each other the friction force can be written as

$$
\mathbf{F}_{\mathrm{fr}}=-F_{\mathrm{frMax}} \frac{\mathbf{v}_{i j}}{\left|\mathbf{v}_{i j}\right|},
$$

where $\mathbf{v}_{i j}=\mathbf{v}_{i}-\mathbf{v}_{j}-$ a velocity of the particle $i$ relate to particle $j$.

The common friction force acting on the particle is the vector sum of the forces in each contact.

\section{Resistance force in medium}

For an accurate description of the continuous medium between the particles is necessary to use one of the grid method. Obviously, the mesh cells must be much smaller than the size of particles to adequately describe the continuous medium, such as near the points of contact of the particles. Thus, the main computational loading will account for on the calculation of interparticle continuous medium, what considerably limit the possible number of particles in the simulation.

Below, for a more detailed study of the behavior of particles a simplified description of interparticle medium is used taking into account only the change in viscosity with the concentration of the dispersed phase.

Resistance force of a particle in a medium is calculated by Stokes' law [Batchelor, 1967]:

$$
\mathbf{F}_{\mathrm{st}, i}=-6 \pi \mu r_{i}\left(\mathbf{v}_{\mathrm{p}, i}-\mathbf{v}_{1}\right),
$$


where $\mu=\mu(C)$ - dynamic viscosity of fluid (eg, for water under normal conditions $\mu=0,001 \mathrm{~Pa} \cdot \mathrm{s}$ ); $\mathbf{v}_{\mathrm{p}, i}-$ particle velocity; $\mathbf{v}_{1}-$ liquid velocity; $C=1-\varphi-$ the average volume concentration of the particles; $\varphi$-average porosity.

Equation (7) is valid only for single particles at low Reynolds numbers. Because the model assumes the presence of many particles, then the correction of Dougherty-Krieger for the viscosity is used [Volkov, 2001; Krieger, Irvin, 1972]

$$
\mu=\frac{\mu_{0}}{\left(1-\frac{C}{0.6}\right)^{\frac{3}{2}}} .
$$

\section{Boundary and initial conditions}

The computational domain is a rectangular parallelepiped with the base 1 by 1 length units (for different number system it can be any physical length, e.g. $1 \mathrm{~mm}$ ) and the required height.

Since the problem is solved in terms of discrete element method, the boundaries of the container is parts of the surface of pseudoparticles with radii $r=r_{b} \gg 1$, (where $r_{b}-$ a constant determined from the condition that in the considering parallelepiped a deviation of the sphere surface from the plane is small, for example, $\left.r_{b}=1000\right)$ and coordinates $\mathbf{x}_{\mathrm{b} i}(i=1 \ldots 5)$ :

$\left(0.5,0.5,-r_{b}\right)$ - top particle,

$\left(-r_{b}, 0.5,0.5\right)-$ left particle,

$\left(r_{b}+1,0.5,0.5\right)$ - right particle,

$\left(0.5,-r_{b}, 0.5\right)$ - back particle,

$\left(0.5, r_{b}+1,0.5\right)$ - front particle.

Conditions at these boundaries are formulated as follows:

$$
\begin{aligned}
& x_{b i}=\text { const }, i=1 \ldots 5, \\
& \left|v_{b i}\right|=0, i=1 \ldots 5, \\
& \left|F_{f r}\right|_{x=0, x=1, y=0, y=1}=0, i=2 \ldots 5 .
\end{aligned}
$$

The last condition in (9) means that the frictional force on the boundaries 2-5 equals to zero (this condition should be used if necessary to eliminate the influence of the side boundaries).

The condition on the top of computational parallelepiped is determined in accordance with the feature of a given task.

These are cases of the simulations below:

a) layer formation as the result of sedimentation monodisperse and bidisperse suspensions;

b) consolidation of the filtering layer by a piston.

The boundary conditions corresponding to these processes will be described in conjunction with the results.

Initial conditions for the set of particles are:

$$
\begin{aligned}
& \mathbf{x}_{i, \text { initial }}=\text { random, } \\
& \mathbf{v}_{i, \text { nitial }}=0,
\end{aligned}
$$

where random - a real random number between 0 and 1 with uniform distribution low. The initial coordinates of particles are although determined randomly, but the condition is checked for nonintersection of particles for any $i$ and $j$ :

$$
\left|\mathbf{x}_{i}-\mathbf{x}_{j}\right|>r_{i}+r_{j}
$$




\section{Numerical realization}

To solve the above problem the system of equations (1) integrates numerically with counting the relations (2)-(8), primary (10), (11) and boundary (9) conditions.

Since the system (1) must be solved for each particle and in general each particle can interact with each, so the task for the system of $N$ particles has complexity of order $N^{2}$. (It means the number of operation is proportional tom $N^{2}$ ). Using a spatial decomposition (partition) of the computational domain, it's possible to reduce the complexity of the problem, because the particles will interact only with those particles that are in the same cell decomposition, thus there is need for data transfer between cells and the implementation mechanism of a particle migration from one cell decomposition to another. An additional significant reduction of computation time can be achieved reached by parallel processing of particles in each cell on multiprocessor computers.

The following results were obtained on a cluster computer SKIF-Cyberia (performance 12 TFlops), using a program written in C++ library and MPICH for parallel computing.

As a test problem the system of 10 particles falling serially to the bottom of the container was considered. At the initial time the particles are strictly one above the other at a distance of $0.1 \mathrm{~mm}$ along the vertical axis $Z$. Obviously the particles will fall down forming a vertical column because there are no forces that would be displace the particle in $\mathrm{X}$ and $\mathrm{Y}$ directions.

In this example, taken: particle size $d=0.1 \mathrm{~mm}$, density $\rho=2500 \mathrm{~kg} / \mathrm{m}^{3}$ (sand), Young's modulus $E=100 \mathrm{GPa}$, and Poisson's ratio $v=0.2$ (silicon), the coefficients of adhesion and friction are set to zero, $\mu=1 \cdot 10^{-3} \mathrm{~Pa} \cdot \mathrm{s}$ (water), $B=1$.

The dependence of join deformation of the 1st and 2nd particles $\left(\frac{h}{d}=\frac{d-\left|\vec{x}_{2}-\vec{x}_{1}\right|}{d}\right)$ on time is shown at Fig. 3a. Every subsequent particles fall on the column with a period $T \approx 0.075 \mathrm{~s}$. Every time when the next particle touch the column the oscillations occurs due to the convertation of kinetic energy to the potential elastic energy.

There is the detailed picture of the deformation in contact of 1 st and 2nd particle occurred as a result of the fall of $3^{\text {rd }}$ particle on column of the first two particles at Fig. 3b. highlighted by the dotted line.

The relative position of two particles is characterized by fluctuations, which decay due to the viscosity of the medium among the particles (Fig. 3b). Without viscosity $(\mu=0)$ particle bounces after the fall and the height of the rebound remains constant, i.e. total energy is conserved, and the computation scheme hasn't numerical viscosity. Minor oscillations in the system of 10 particles remain in time from $t=0.71 \mathrm{~s}$ and up to the end of the simulation; probably these variations are due to numerical errors which is also noted in [Siiriae, Jouko, 2007].

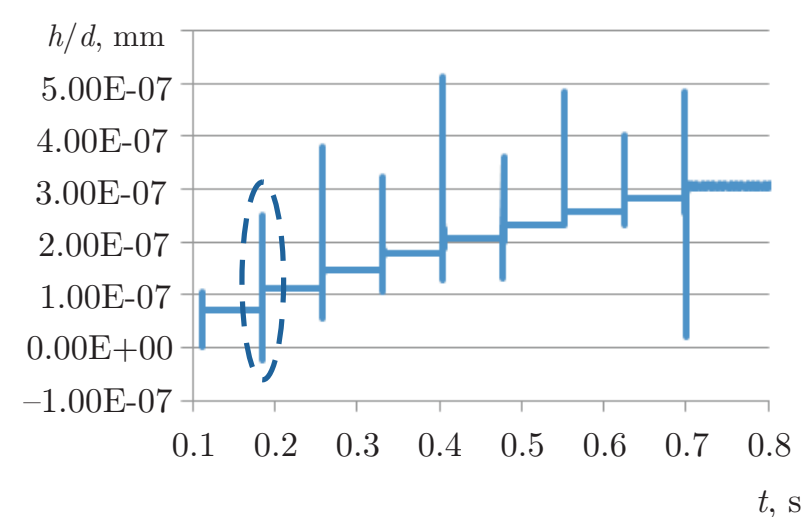

(a)

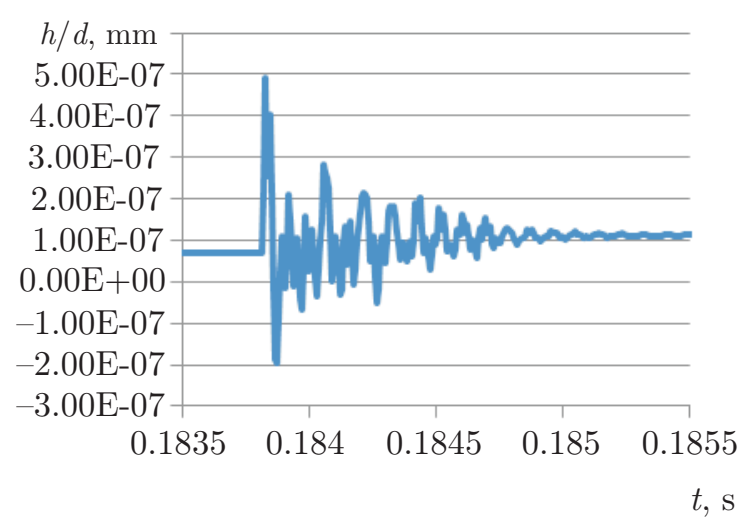

(b)

Fig. 3. The dependence of join deformation of the $1^{\text {st }}$ and $2^{\text {nd }}$ particles on time (a) and the detailed picture of the deformation occurred as a result of the fall of $3^{\text {rd }}$ particle (b) 
The weight effecting on the $1^{\text {st }}$ particle from the overlaying 9 particles can be defined analytically: $F=9 m g \approx 1.15 \cdot 10^{-7} H$. This weight leads to the relative join deformation of $1^{\text {st }}$ and $2^{\text {nd }}$ particles due to the solution of the Hertz problem: $h / d \approx 3.0 \cdot 10^{-7}$. This magnitude is in good agreements with the computed one (Fig. 3a).

Priori underestimate of the characteristic time of interaction of particles is based on the fact that the average velocity of the particle in the interaction is less than the velocity of the particle before the interaction, which can be token as steady-speed particle according to Stokes (7): $v=2 r^{2} \Delta \rho g / 9 \mu$. According to the energy conservation law if the kinetic energy of the particle is completely converted into elastic energy it is possible to determine the maximum deformation $h_{\max } \approx r^{\frac{13}{5}} \Delta \rho^{\frac{6}{5}} g^{\frac{4}{5}} / 5 \mu^{\frac{4}{5}} K^{\frac{2}{5}}$. Then the characteristic time of interaction between the particles is $t^{*}=h_{\max } / v \approx \sqrt[5]{r^{3} \Delta \rho \mu / K^{2} g}$. This is $t^{*} \approx 3 \cdot 10^{-7} \mathrm{~s}$ for above-mentioned physical parameters.

It is clear that in order to correctly calculation the time step must be chosen much shorter than the characteristic period of elastic vibrations (in the example in Fig. $4 \mathrm{~b}$ about $10^{-5} \mathrm{~s}$ ). In all the calculations, this step is automatically selected during the computation from the condition (derived from the same considerations that $\left.t^{*}\right): \Delta t=k_{t} r \sqrt[5]{\frac{25 \pi^{2} \Delta \rho^{2}}{9 K^{2} v_{\max }}}$, where $v_{\max }$ - the maximum velocity of all particles, $k_{t}$ - dimensionless coefficient of the calculation scheme $\left(0<k_{t}<1\right)$.

It is known that the stress wave is spreading at a speed of sound in the medium so for accurately describing the propagation of stress waves and displacement a step of time integration must be defined from the condition than the shift of the stress wave is not more than one diameter of the smallest particles from the existing set. Otherwise, the velocity of wave propagation in the model will be lower than the physical speed of sound in the solid matter.

As shown above the simulation includes a wide time scales range of the processes: from the micro-level interaction between individual particles (about $10^{-6} \mathrm{~s}$ ) up to the macro-level effects of shrinkage and creep, which in practice can be occurring during days (about $10^{5}$ ). This wide range of time scales (11 orders) makes a big contribution to the simulation complexity.

With a rough estimate, given that the current cluster supercomputer has a performance of several tens of teraflops (operations per second), in real time can be proceeded a system of about 1000 particles. Proceeding of a system of $10^{5}$ particles at time scale $1 \mathrm{~s}$ needs about 3 hours of computer time.

\section{Results of simulations}

Sedimentation in nature and technology often leads to the formation of sediment (silt in water reservoirs, precipitation in wastewater treatment plants, etc.). The structure of the sediment is determined by the deposition process, the composition of the dispersed phase and shrinkage characteristics of sediments after the proper sedimentation of particles of the suspension finishes.

Modeling the formation and dynamics of sediment was carried out herewith on the basis of the DEM. The Stated below results were obtained with the following values of physical constants: 2000 particles with a radius of $0.05 \mathrm{~mm}$, the width of the container $1 \mathrm{~mm}$, initial height of layer $10 \mathrm{~mm}$, the initial volume concentration of the suspension $C=0.1$, Young's modulus $E=100 \mathrm{GPa}$, and Poisson's ratio $v=0.2, k_{f r}=0.1, K_{a d}=0.1 \mathrm{~N} / \mathrm{mm}, \rho=2500 \mathrm{~kg} / \mathrm{m}^{3}, \mu=1 \cdot 10^{-3} \cdot \mathrm{Pa} \mathrm{s}, B=0.9$.

\section{Sedimentation of bidisperse suspension}

At Fig. 4 the chronogram of the particle sedimentation are presented at moments $0,0.5,4,8 \mathrm{~s}$.

At the initial time the concentration of particles is approximately uniform by height; since half a second of the particle deposition process a small sediment layer has formed (in frame of the numeri- 
cal simulation we will call "sediment layer" a system of particles which have many contacts between each other - the number of contacts for each particle is greater than 5). At time $4 \mathrm{~s}$, the deposition process is completed; it's visually noticeable that the upper layer is less dense than the lower, which was compressed by overlying particles. Then a layer continues to slowly sit down $4 \mathrm{~s}$ more.

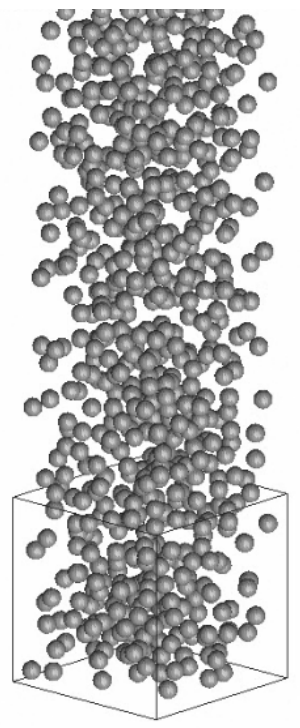

(a) $t=0 \mathrm{~s}$

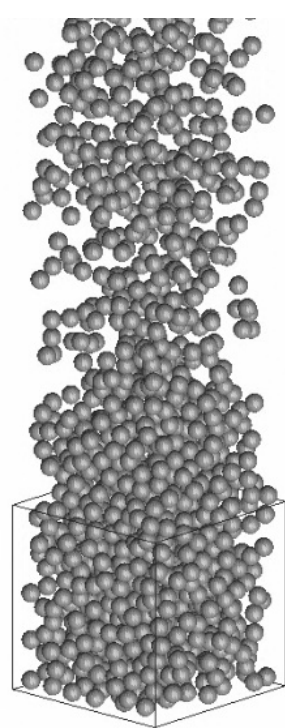

(b) $t=0.5 \mathrm{~s}$;

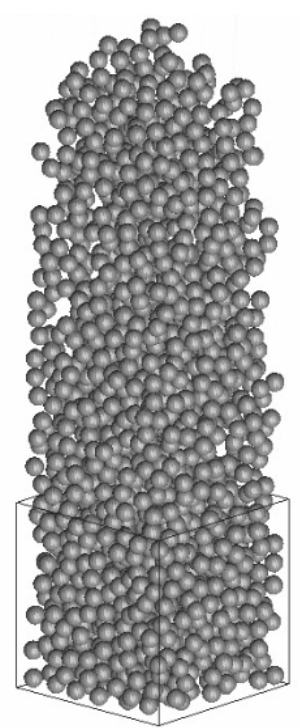

(c) $t=4 \mathrm{~s}$;

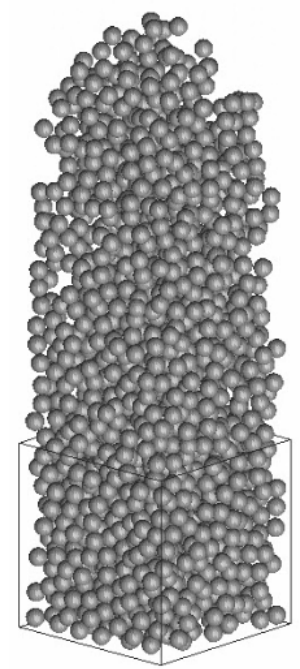

(d) $t=8 \mathrm{~s}$

Fig. 4 The chronogram of the particle sedimentation at moments $0,0.5,4$ and $8 \mathrm{~s}$

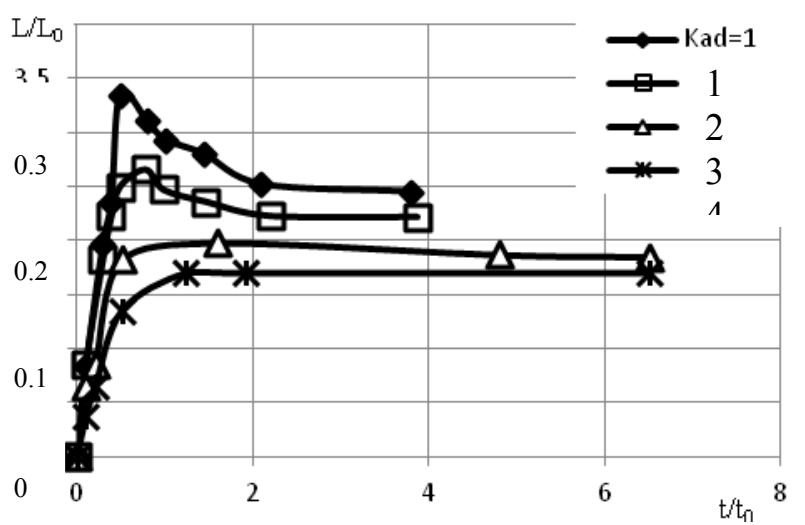

(a) simulation; $1-K_{\mathrm{ad}}=1,2-K_{\mathrm{ad}}=0.5$, $3-K_{\mathrm{ad}}=0.1,4-K_{\mathrm{ad}}=0.01$

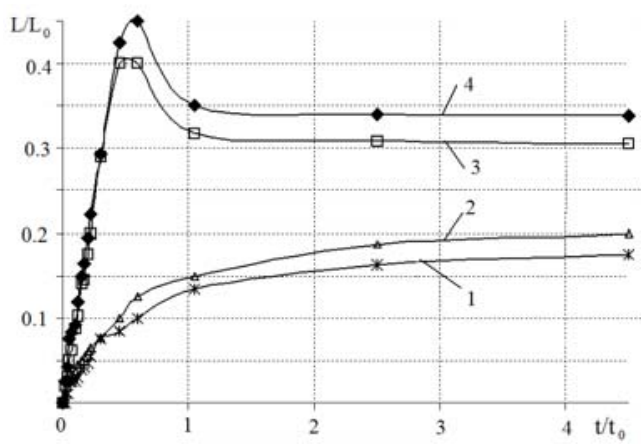

(b) experiment [Dueck, Purevjav, Kilimnik, 2004]; concentration $\mathrm{MgCl}_{2}$ : $1-10^{-1} \mathrm{~mol} / \mathrm{l}, 2-10^{-2} \mathrm{~mol} / \mathrm{l}$, $3-10^{-3} \mathrm{~mol} / \mathrm{l}, 4-10^{-4} \mathrm{~mol} / \mathrm{l}$.

Fig. 5. The dependence of dimensionless height of sediment layer on the dimensionless time for different magnitudes of the adhesion forces between particles

Fig. 5a shows the results of modeling the growth dynamics of sediment over time, as in Fig. 5bthe corresponding results of the laboratory experiment [Dueck, Purevjav, Kilimnik, 2004]. Layer height is measured in fractions of the initial height of the suspension; dimensionless time is time divided by deposition time of a single particle according Stokes: $t_{0}=9 \mu L_{0} / 2 r^{2} \Delta \rho g$.

In the experiments, the level of adhesive force varies due to changes in the $\mathrm{MgCl}_{2}$ solution. According to [Dueck, Purevjav, Kilimnik, 2004] change in the concentration of $\mathrm{MgCl}_{2} 10^{-4} \mathrm{~mol} / 1$ to $10^{-1}$ $\mathrm{mol} / \mathrm{l}$ varies the adhesive force of nearly 100 times. It can be seen that there is a qualitatively similarity of results in experiments and calculations. Quantitative agreement with experimental results cannot be 
achieved due to the fact that there are a few orders more particles with much less size in the experiment than in the calculation.

A layer of contacting particles with porous unstable structures is forming during the relatively rapid sedimentation. This instability is the stronger the higher $K_{\text {ad. }}$.

The phase of shrinking followed after sedimentation occurs due to the slow movement (friction force inhibits this movements) of searching stable positions particles.

The obtained in the simulations dependence of the minimum porosity attained at $t \gg t_{0}$ on the adhesion coefficient obeys the equation: $\varphi-\varphi_{0} \approx 0.21 \cdot K_{\text {ad }}{ }^{0.53}$, where $\varphi_{0}=0.4$; and the dependence of $1 /\left(\varphi-\varphi_{0}\right)$ on $1 / K_{\mathrm{ad}}$ is linear function: $\frac{1}{\left(\varphi-\varphi_{0}\right)} \approx 0.77 \frac{1}{K_{\mathrm{ad}}}-3.35$. The linearity of $1 /\left(\varphi-\varphi_{0}\right)$ on $1 / K_{\mathrm{ad}}$ is followed from the theoretical consideration and proved by the experiments [Dueck, Purevjav, Kilimnik, 2004].

\section{Case of bidisperse suspension}

In the following calculations, all particles have one of the two sizes $r_{1}=0.0025 \mathrm{~mm}, r_{2}=0.05$ $\mathrm{mm}$, thus $r_{1} / r_{2}=2$. Initial height of suspension $-10 \mathrm{~mm}$, the initial volume concentration of fine and coarse particles are equal and is $C_{1}=C_{2}=2.5 \%$, and the distribution of fine and coarse particles at the initial time is taken uniform throughout the volume. Other parameters remain the same.

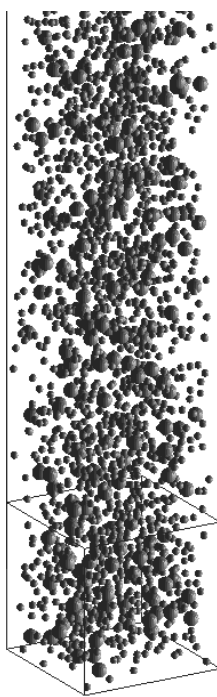

$0 \mathrm{~s}$

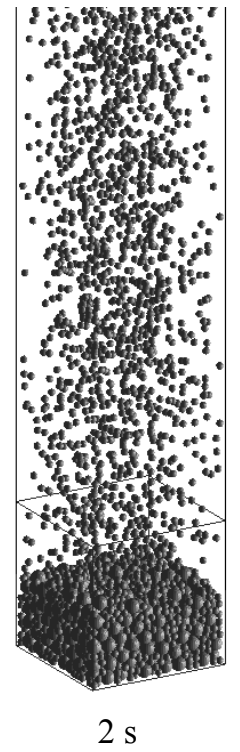

(a)

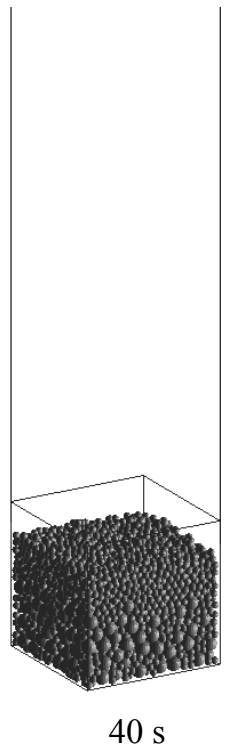

$40 \mathrm{~s}$

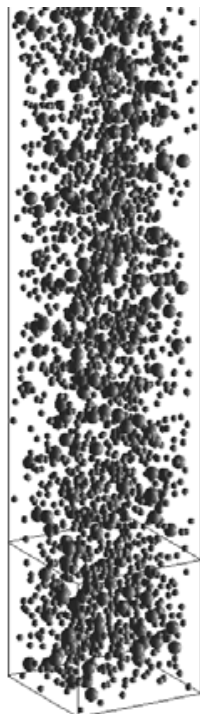

$0 \mathrm{~s}$

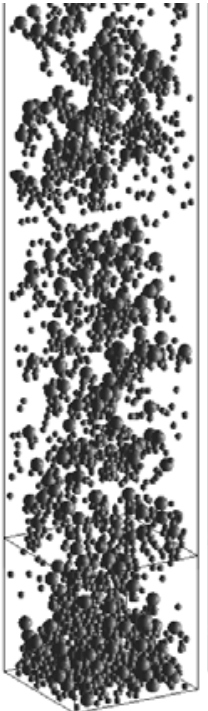

$1.7 \mathrm{~s}$

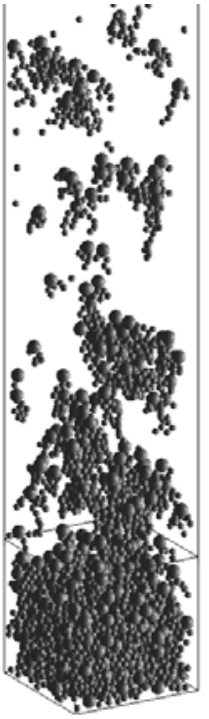

$8.7 \mathrm{~s}$

(b)

Fig. 6 The state of sedimentation process at different times: (a) $K_{\mathrm{ad}}=0 \mathrm{~N} / \mathrm{mm}$, (b) $K_{\mathrm{ad}}=0.1 \mathrm{~N} / \mathrm{mm}$

In Fig. 6 the state of the process of sedimentation at different times is visualized for cases $K_{\text {ad }}=0 \mathrm{~N} / \mathrm{m}$ and $K_{\text {ad }}=0.1 \mathrm{~N} / \mathrm{mm}$.

It is evident that in the case $K_{\text {ad }}=0.1 \mathrm{~N} / \mathrm{mm}$ initially homogenous suspension in the process of sedimentation becomes significantly heterogeneous - the particles form flakes. Similar effects have been observed in experiments [Hogg, 2000; Obiakor, 1969]. Noteworthy that there isn't the coagulation in the suspension in the case if the particles have the same size that can be seen from Fig. 4.

The formation of flakes, as shown in Fig. 6b, can be explained as follows. Large particles according the Stokes' law are moving faster than small ones, "sweeping" them on their way. And because there is the adhesive force between the particles, the small particles stick to big ones, and they continue to move as a whole collecting along the way more and more small particles. In the case of 
a monodisperse suspension of the effect of flakes formation will not be presented due to the fact that all particles move at the same velocity until the touch the lower boundary of the computational domain or the layer of already deposited particles.

Fig. 7 shows the concentration distribution by height of fine and coarse fractions at a time when all the particles have settled.

There is only a slight separation of the layer by sediment fractions in case of $K_{\text {ad }}=0.1 \mathrm{~N} / \mathrm{mm}$, probably, due to the fact that at some point of time the small and large particles have formed flakes and moved as an unit with the same speed. If $K_{\mathrm{ad}}=0 \mathrm{~N} / \mathrm{mm}$ there is a significant separation of fractions along the height layers: large particles are in the bottom, small ones in the top part. These results agree qualitatively with the experimental data [Purevjav, 2006].

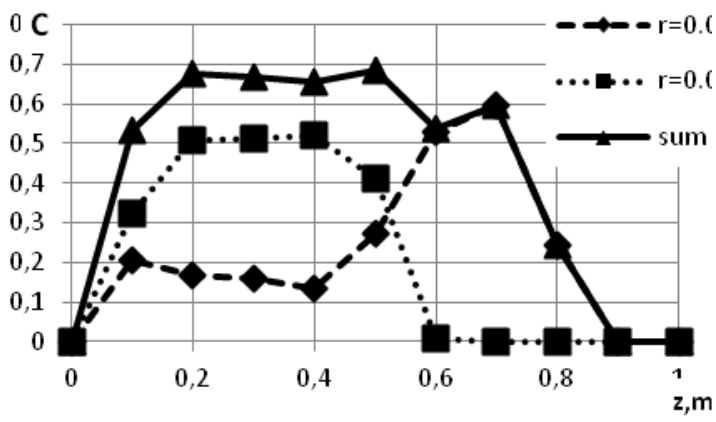

(a)

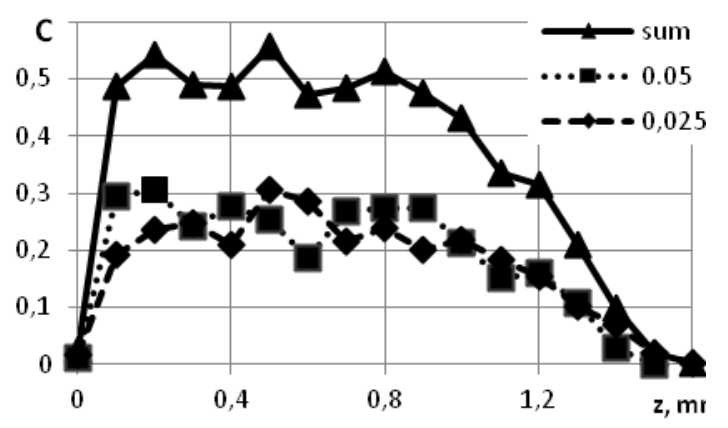

(b)

Fig.7. The concentration distribution by height of fine and coarse fractions at a time when all the particles have settled: (a) $K_{\text {ad }}=0 \mathrm{~N} / \mathrm{mm}$, (b) $K_{\text {ad }}=0.1 \mathrm{~N} / \mathrm{mm}$

There are significant stochastic fluctuations of curves in this and subsequent figures due to random initial conditions of the system and a small amount of particles because each calculation is carried out on a computer long enough time (several days), and as a consequence, there is no opportunity to carry out and averaging the data obtained from a few realizations of the numerical experiment with different initial conditions.

\section{Case of polidisperse suspension}

Sedimentation of polydisperse suspension demonstrates the same characteristics as mono- and bidisperse cases: sedimentation is accompanied by formation of a layer and its subsequent shrinkage. Segregation of the particle by size and formation of flakes in suspension are observed in the layer if there is adhesion. Therefore, the presentation of the results here will be limited to an illustration of the nature of particle motion in a layer at the stage of shrinkage.

The mechanism of shrinkage of the layer at the micro level is illustrated in Fig. 8, which shows the visualization of the shrinkage of polydisperse layer of particles at different times. At some moment since the beginning of shrinkage four particles are highlighted and their subsequent trajectories are tracked over time.

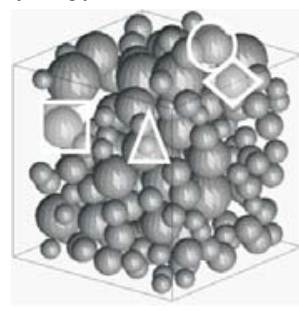

1

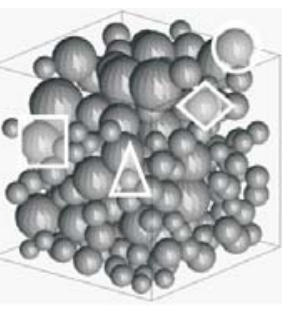

2

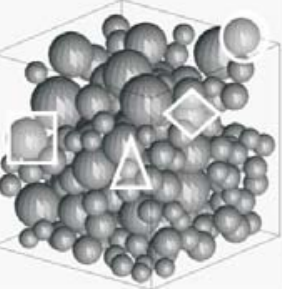

3

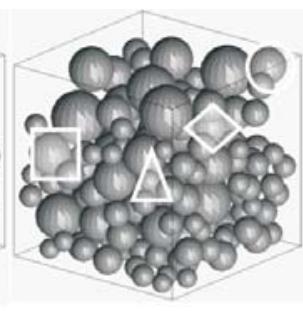

4

Fig. 8. Position of the particles in moments: $1-t / t_{0}=1 ; 2-t / t_{0}=1.5 ; 3-t / t_{0}=2 ; 4-t / t_{0}=2.5$ 
The figure shows that over time both absolute and relative positions of the particles changes, and the layer shrinkage occurs due to these micromotions. It is seen that during this period of time, each of the selected particles moved (not necessarily in a downward direction) at a distance of about one particle size. In general, during the reporting period the porosity of the layer decreased by about $10 \%$. Noticeably there is predominance of small particles at the bottom of the container.

\section{Consolidation of the filtration layer}

Compaction the filtration layer in practice is a necessary stage in the general process of filtering. This is done purposefully to dehydrate the dispersed material and often compaction is a process that determines the quality of the product (e. g., paper). During compaction (consolidation) of the layer by external device (piston, rolls) the significant pressure acts on the particle forcing the particles to move relative to each other. The water from the pore space is squeezed out, and the particles form a dense packing.

To simulate the process of compression the additional six pseudoparticles is used (additional boundary condition), which locates on top of the computational domain and presses on the underlying particle with a certain pressure. The initial position of the particle is set equal to $\left.\mathbf{x}_{\mathrm{b} 6}\right|_{t=0}=r_{\mathrm{b}}+H$, where $H$ - initial height of the piston.

The pressure drop is calculated as the difference between the defined pressure of piston, the pressure at the filter output and pressure, which the particles of layer are provided on the piston: $\Delta P=P_{\text {press }}-P_{0}-P_{\text {part }}$, where $P_{\text {press }}-$ constant pressure of the press, $P_{0}$ - the pressure at the filter output, which is assumed to be zero: $P_{0}=0 \mathrm{~Pa}$. The pressure of the particles on the piston is given by: $P_{\text {part }}=\sum_{j=1}^{N_{\mathrm{p}}} f_{\mathrm{p} j} / S_{\text {press }}$, where $N_{\mathrm{p}}$ - the number of particles, which are in contact with the piston, $f_{\mathrm{p} j}-$ the force of elastically interaction of particles with the piston, $S_{\text {press }}-$ piston area.

The initial condition for the piston velocity in case of movement with constant pressure is defined from the Darcy's law [Nigmatulin, 1978]: $v_{1}=k_{\mathrm{D}} \frac{\Delta P}{\mu h_{\mathrm{L}}}$, where $v_{1}$ - velocity of liquid and therefore the velocity of the piston, $\Delta P$ - pressure drop on the layer $h_{\mathrm{L}}$ (the height of the layer in that case), $\mu$ - liquid viscosity, $k_{\mathrm{D}}$ - Darcy permeability coefficient, which for the bulk layer of particles of radius $r$ and porosity of the layer $\varphi$ is determined according to Kozeny-Carman: $k_{\mathrm{D}}=\frac{r^{2} \varphi^{3}}{37.5 \cdot(1-\varphi)^{2}}$.

Since the filtration of fluid takes place not only through the layer of particles, but also through the filter material located on the lower boundary of the computational domain, then the effective permeability coefficient for such a system can be written as: $\frac{h_{\mathrm{L}}}{k_{\text {ef }}}=\frac{h_{\mathrm{L}}}{k_{\mathrm{D}}}+\frac{h_{\text {filter }}}{k_{\text {filter }}}$, where $k_{\text {filter }}-$ coefficient of filtration referring to the bottom of the filter. Then Darcy's law can be written as $v_{1}=\frac{k_{\mathrm{D}} k_{\text {filter }}}{h_{L} k_{\text {filter }}+h_{\text {filter }} k_{D}} \frac{\Delta P}{\mu}$.

For the calculations we used the following parameters: 2000 particles with a radius of $0.05 \mathrm{~mm}$, the width of the container $1 \mathrm{~mm}$, initial height of layer $10 \mathrm{~mm}$, the initial volume concentration of suspension $C=0.1$, Young's modulus $E=100 \mathrm{GPa}$, and Poisson's ratio $v=0.2, k_{\mathrm{fr}}=0.1, K_{\mathrm{ad}}=0.1 \mathrm{~N} /$ $\mathrm{mm}, \rho=2500 \mathrm{~kg} / \mathrm{m}^{3}, \mu=1 \cdot 10^{-3} \cdot \mathrm{Pa} \mathrm{s}, B=0.9$, the pressure piston $P_{\text {press }}=1 \mathrm{MPa}$.

Fig. 9 shows the visualization of the layer compression by piston. The dynamics of the piston and the dependence of the particle-fluid pressure on the piston are shown in Fig. 10. It is seen that at time $3.5 \mathrm{~s}$, the piston has touched sediment layer, what is reflected in the fact that the amount of the 
contact particles with the piston increases quickly. After that the backpressure of the particles on the piston increases during the compression layer.

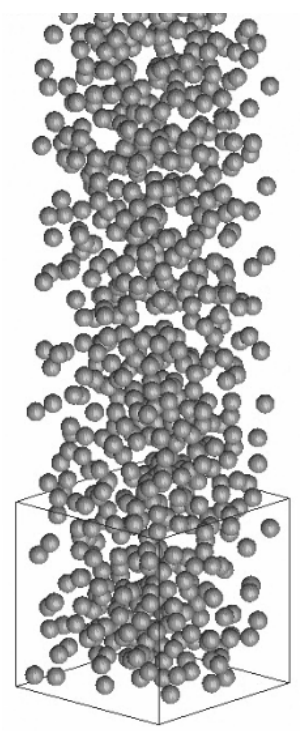

(a) $\quad t=0 \mathrm{~s}$;

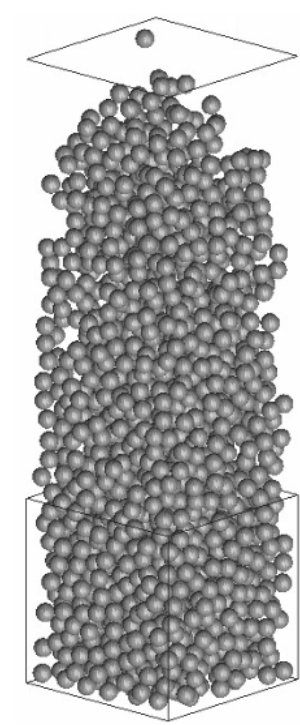

(b)

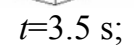

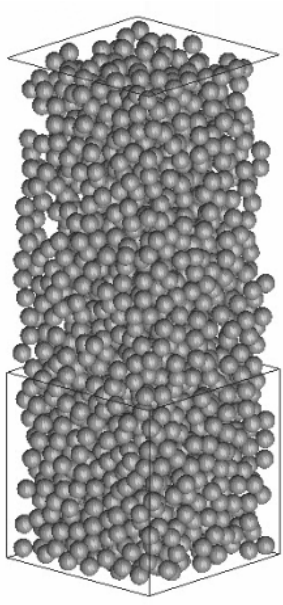

(c)

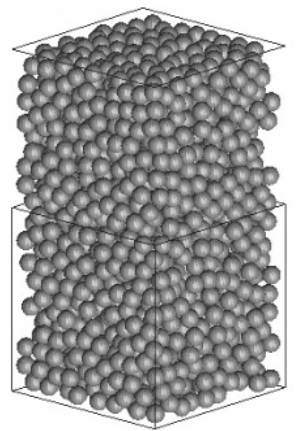

(d) $\quad t=8 \mathrm{~s}$;

Fig. 9. The chronogram of filtering process

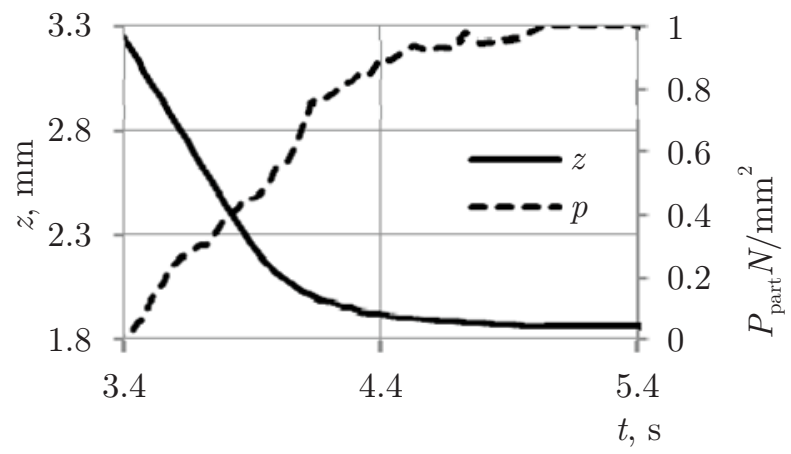

Fig. 10. The dynamics of the piston and the dependence of the particle-fluid pressure on the piston

Fig. 11 shows an example of the dependence of the layer density distribution (one numerical realization) and the pressure of the layer on height at different times. As a layer pressure we suppose a ratio of the sum of all elastic forces acting on the particle by other particles to the surface area of the particle.

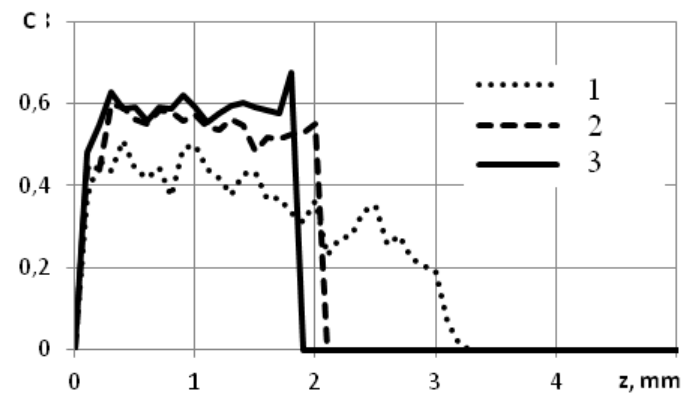

(a)

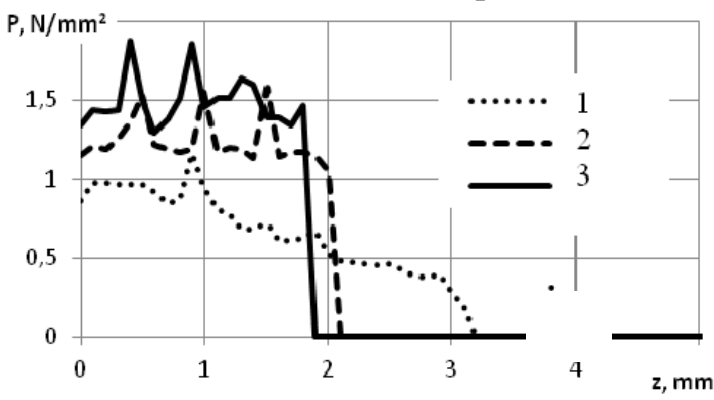

(b)

Fig. 11. The distribution of the particle concentration through the layer (a) and pressure ob particles (b) by layer height in different time moments: $1-t=3 \mathrm{~s}, 2-t=5 \mathrm{~s}, 3-t=8 \mathrm{~s}$ 
The figures show that at time $3 \mathrm{~s}$, before the press began to effect on a layer, the corresponding parameters - the density and pressure - decreasing with increasing of height. After the filtering process both parameters were approximately monotonic, the average density of the layer increased by approximately 2 times in comparison with the uncompressed state.

\section{Discussions}

The proposed numerical model of sedimentation and filtering of the suspension includes the phenomenon of particles sedimentation, microinteraction of particles, the formation of mesostructures (pores, arches, etc.), shrinkage and compaction of sediment layer, flocculation.

Formulated mathematical model based on the discrete elements method, which solved the dynamic problem of motion of individual particles in a collective.

Within the scope of implementation of the model one must take into account the fact that only limited number of particles (about $10^{3}$ ) can be involved in the simulation and only small computational domain of a few millimeters can be processed. Length of the edge considered here container exceeds only about 10-20 times of the particles size. It is known that the influence of near-wall effects on the structure of packing propagate for approximately 2-4 particle size [Dueck, Dyachenko, Minkov, 2006; Neesse, Dueck, Djatchenko, 2009]. This thickness of simulated domain can be minimized by formulating the absence of friction on the wall, such a computational domain is to be considered as representative volume of the whole simulating region.

DEM today cannot replace the simulation of sedimentation and filtration by methods of continuum mechanics [Zhuzhikov, 1971; Wakeman, Tarleton, 1999; Nigmatulin, 1978], but it may provide some details of these processes, especially those who are directly dependent on interaction between the particles through the surface physical and chemical phenomena and friction. Some of these phenomena, such as shrinkage, formation of aggregates (flakes), etc have been simulated and shown above. In addition, this approach allows one to visualize these processes and provides data that are difficult to obtain from experiments, for example, the pressure distribution in a sediment layer or filtration layer.

Among the obvious disadvantages it should be noted extremely high requirements of the model for computing resources: even modern cluster computers cannot process number of particles that are present in real physical units; in addition, high resource consumption limits the possibility of parametric studies in a wide range of parameters.

\section{Conclusions}

Possibilities of the discrete elements are illustrated; its limits and conditions of use are shown.

It is shown that, in agreements with the experiments, the formation of the layers during the deposition depends on the surface (adhesion) forces and the size distribution of the suspension. It's illustrated that if the adhesion is strong enough the shrinkage of the layer occurs after the sedimentation leading to a nonmonotonic dynamics of its height. At low adhesion the height of the forming layer tends to its end value monotonically.

It's noted that there is the phenomenon of flakes formation in settling nonmonodisperse suspension if there exists-adhesion. It is shown that the adhesion prevents segregation in the layer of the particle by size. The character of movement of particles, leading to the formation of stable packages is shown on an example of the simulation of polidisperse particles shrinkage.

The slowing down movements of the piston under resistance from the particles and the formation of a density homogeneous (except for the top region) layer is illustrated by the simulation of compression of the filtration layer. 


\section{Legends}

$A$ - Hamaker's constant, a - acceleration, J; $B$ - energy restitution coefficient; $C$ - average volume concentration; $c_{\infty}$ - ions concentration in liquid away from particle surface; $d$-diamets of particle, $\mathrm{m} ; e$ - electron charge, $\mathrm{Cl} ; E$ - Young's modulus, $\mathrm{Pa} ; \mathbf{f}$ - elastic interaction force between two particles, $\mathrm{N} ; f$ - magnitude of interaction force, $\mathrm{N} ; \mathbf{F}$ - resulting force acting on the particle, $\mathrm{N} ; \mathbf{F}_{\text {ad }}-$ adhesion force between two particle in contact, $\mathrm{N} ; \mathbf{F}_{\mathrm{fr}}$ - generalized rolling and sliding friction force, $\mathrm{N} ; \mathbf{F}_{\text {st }}$ - resistance force according Stocks, N; $F_{\text {at }}$ - attractive component of adhesion force due to Van der Waals force, $\mathrm{N} ; F_{\mathrm{r}}$ - repulsive component of adhesion force according Gouy-Chapman theory, $\mathrm{N} ; F_{\text {mov }}-$ moving force, i.e. the result of all force except the friction force in the current contact, N; $F_{\text {frMax }}$ - maximum possible friction force, $\mathrm{N} ; \mathbf{g}$ - gravity acceleration, $\mathrm{m} / \mathrm{s}^{2} ; h$ - penetration depth of particles, $\mathrm{m} ; \mathrm{H}$ - initial height of the piston, $\mathrm{m} ; H_{0}$ - distance between the surfaces of interacting particles (usually the intermolecular distance), $\mathrm{m} ; h_{\mathrm{L}}$ - particle layer height, $\mathrm{m} ; k$-Boltzmann's constant, $\mathrm{J} / \mathrm{K} ; K$ - effective Young's modulus, $\mathrm{Pa} ; K_{\mathrm{ad}}-$ adhesion coefficient, $\mathrm{N} / \mathrm{m} ; k_{\mathrm{D}}$ - Darcy permeability coefficient, $\mathrm{m}^{2} ; k_{\mathrm{ef}}$ - effective Darcy permeability coefficient, $\mathrm{m}^{2} ; k_{\mathrm{f}}$ - coefficient of filtration referring to the bottom of the filter, $\mathrm{m}^{2} ; k_{\mathrm{fr}}$ - effective friction coefficient; $k_{t}$ - coefficient in calculation scheme; $L_{0}$ - initial height of suspension, $\mathrm{m} ; m$ - particle mass, $\mathrm{kg} ; N$ - number of contact of particle; $P$ - pressure, $\mathrm{Pa} ; P_{0}$ - the pressure at the filter output, $\mathrm{Pa} ; P_{\text {part }}$ - pressure of the particles on the piston, $\mathrm{Pa} ; \Delta P$ - pressure drop on the layer, $\mathrm{Pa} ; r$ - particle radius, $\mathrm{m} ; R$ - effective radius of two particles, $\mathrm{m}$; random - a real random number between 0 and 1 with uniform distribution low; $t$ - time, $\mathrm{s} ; t^{*}$ - characteristic time of interaction between the particles, $\mathrm{s} ; T$-temperature, $\mathrm{K} ; \mathbf{v}$ - vector velocity, $\mathrm{m} / \mathrm{s} ; \mathbf{v}_{p}-$ velocity of the particle, $\mathrm{m} / \mathrm{s} ; \mathbf{v}_{l}$ - liquid velosity, $\mathrm{m} / \mathrm{s} ; \mathbf{v}_{i j}-$ velocity of the particle $i$ relate to particle $j, \mathrm{~m} / \mathrm{s} ; W$ - particle energy, $\mathrm{J} ; W_{0}$ - initial particle energy, $\mathrm{J} ; \mathbf{x}$ - particle center position, $\mathrm{m} ; z$-valence of ions in medium; $\varepsilon$ - permittivity of the medium; $\varepsilon_{0}$ - the electric constant, $\mathrm{F} \cdot \mathrm{m}^{-1} ; \Delta \rho$ - the difference between density of matter of particles and interparticle medium, $\mathrm{kg} / \mathrm{m}^{3} ; \Delta t$ - integration step is computational scheme for the time, $\mathrm{s} ; \varphi$ - average volume porosity of particle layer; $\psi_{0}$ - the surface potential of particles, $\mathrm{J} ; \mu$-liquid viscosity, Pa.s; $\rho$-particle matter density, $\mathrm{kg} / \mathrm{m}^{3} ; v-$ Poisson's ratio of particle material, $\kappa$ - reciprocal Debye length, $1 / \mathrm{m}$. Indexes: $b$ - boundary; press - piston, max - maximal.

\section{References}

Ananda A., Curtisa J. S., Wassgrenb C. R., Hancockc B. C., Ketterhagenc W. R. Predicting discharge dynamics from a rectangular hopper using the discrete element method (DEM) // Chemical Engineering Science. - 2008. № 63. - P. 5821-5830.

Batchelor G. K. An Introduction to Fluid Dynamics. Cambridge University Press. 1967. 535 p.

Dueck J., Dyachenko E. N., Minkov L. L. Simulation of random packing of spheres // Physical mesomechanics. - 2006. V. 9. - P. 63-69.

Dueck J., Purevjav D., Kilimnik D. A contribution to the theory of porosity of fine-grained sediments // J. Eng. Phys. Thermophys. - 2004. - V. 77, № 1. - P. 93-102.

Dueck J., Ugov V. I. About modeling of structure of bulk layer// Ing.-Phys. Journal. - 2005. V. 78, № 2. - P. 36-43.

$\operatorname{Hogg}$ R. Flocculation and dewatering. Int. J. Miner. Process. - 2000. № 58. - P. 223-236.

Israelachvili J. Intermolecular and Surface Forces. San Diego: Academic Press. 1995. 450 p. 
Krieger, Irvin M. "Rheology of Monodisperse Latices", Advances in Colloid and Interface Science. 1972. № 3. - P. 111-136.

Kruggel-Emden H., Simsek E, Rickelt S., Wirtz S., Scherer V. Review and extension of normal force models for the Discrete Element Method // Powder Technology. - 2007. - Vol. 171, Iss. 3. P. 157-173.

Landau L. D., Livshic E. M. Elasticity theory. - Moscow: "Nauka", 1987. - 246 pp.

Moysey P. A., Thompson M. R. Modelling the solids inflow and solids conveying of single-screw extruders using the discrete element method// Powder Technology. - 2005. № 153. - P. 95-107.

Neesse Th., Dueck J., Djatchenko E. Simulation of filter cake porosity in solid/liquid separation // Powder Technology. - 2009. № 193. - P. 332-336.

Nigmatulin R. I. Basis of heterogeneous media mechanics. - Moscow: "Mir", 1978. - $336 \mathrm{c}$.

Obiakor E. K., Whitmore R. L. Settling Phenomena in Flocculated Suspension // Rheologica Acta. 1969. № 6. - P. 353-359.

Purevjav D. Packing characteristics of fine-grained filter cake and sediment. PhD. Dissertation. University Erlangen. Der andere Verlag. 2006. 119 p.

Siiriae S., Jouko Y. Particle packing simulation based on Newtonian mechanics // Powder Technology. -2007 . № 174. - P. 82-92.

Timoshenko S., Godier J. N. Theory of elasticity. McGRAW-HILL BOOK COMPANY, 1951. $519 \mathrm{p}$.

Volkov V. A. Colloidal chemistry. Moscow: MSTU. 2001. 640 p.

Vu-Quoc L., Zhang X., Walton O. R. A 3-D discrete-element method for dry granular flows of ellipsoidal particles // Comput. Methods Appl. Mech. Engrg. - 2000. № 187. - P. 483-528.

Wakeman R. J., Tarleton E.S. Filtration: equipment selection, modeling and process simulation. Great Britain: Elsevier Advanced Technology. 1999. 446 p.

Zhu H. P., Zhou Z. Y., Yang R. Y., Yu A. B. Discrete particle simulation of particulate systems: A review of major applications and findings // Chemical Engineering Science. - 2008. Vol. 63, Iss. 23. - P. 5728-5770.

Zhu H. P., Zhou Z. Y., Yang R. Y., Yu A. B. Discrete particle simulation of particulate systems: Theoretical development // Chemical Engineering Science. - 2007. V. 62, Iss. 13. - P. 3378-3396.

Zhuzhikov V. A. Filtering: theory and practice. Chemestry. 1971. 441 pp. 